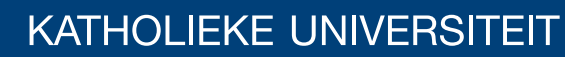 \\ LEUVEN
}

Faculty of Business and Economics

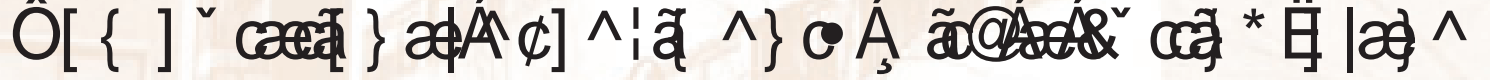
DORLUKP IIRUDMP HLQGH HGIRIP XOURQ

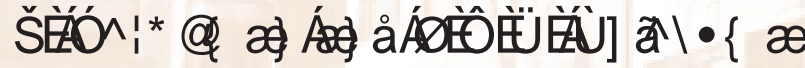

DEPARTMENT OF DECISION SCIENCES AND INFORMATION MANAGEMENT (KBI) 


\title{
Computational experiments with a cutting-plane algorithm for a time-indexed formulation
}

\author{
L. Berghman, F.C.R. Spieksma \\ ORSTAT, K.U.Leuven, Naamsestraat 69, 3000 Leuven, Belgium
}

\begin{abstract}
We consider a time-indexed formulation for the unrelated parallel machine scheduling problem. We show that all polyhedral knowledge known from the single machine problem (in particular, valid inequalities) is applicable to this formulation. We also present new valid inequalities, and we implement a basic cutting-plane algorithm based on these inequalities. Its performance is tested by running it on randomly generated instances.
\end{abstract}

Keywords: unrelated machine scheduling, time-indexed formulation, valid inequalities, cutting plane algorithm.

\section{Introduction}

The time-indexed formulation for single machine scheduling problems is well studied in the literature. Seminal work of Dyer and Wolsey (1990) and Sousa and Wolsey (1992), and further work by Crama and Spieksma (1996) and van den Akker et al. (1999) have resulted in a large body of polyhedral results for the time-indexed formulation. Generally speaking, the major advantage of a time-indexed-formulation is the tight LP-bound, while the greatest disadvantage are the large number of variables, especially when processing times are large. One possible avenue to, at least partially, overcome this difficulty is using column generation, as was done in van den Akker et al. (2000) and Bigras et al. (2008). An arc-time indexed formulation is an extended formulation that yields strictly better bounds that the timeindexed formulation at the cost of an even larger number of variables, one for each pair of jobs and each possible completion time (see, e.g. Sourd 2009; Tanaka et al. 2009).

As far as we are aware, all this polyhedral knowledge has not been applied to timeindexed formulations of scheduling problems with multiple machines, in particular unrelated parallel machine scheduling. This is confirmed by Unlu and Mason (2010) who evaluate integer programming formulations for parallel machine scheduling and recommend to use a time-indexed formulation when job processing times are small. Moreover, they explicitly suggest to develop valid inequalities.

This paper deals with the time-indexed formulation of the unrelated parallel machine scheduling problem, where the processing cost of a job is an arbitrary function of its starting time. Notice that this allows to model many objective functions such as (weighted) sum of completion times, makespan, and to incorporate features such as release times and precedence 
relations. Our goal is to (1) point out that all polyhedral knowledge existing for singlemachine problems can be applied to multi-machine problems, (2) to describe a new class of inequalities for the time-indexed formulation for multiple machines, and (3) to show the computational performance of basic cutting plane algorithm by testing this algorithm on randomly generated instances. Here, we are primarily interested to see to what extent the inequalities bridge the gap between the LP-relaxation and the integer optimum.

The problem statement and the proposed single machine scheduling formulation are presented in Section 2. We show in Section 3 that existing valid inequalities can be applied to our formulation. In Section 4, we present a new class of valid inequalities. Section 5 describes the outcome of running a basic cutting-plane algorithm on randomly generated instances, while Section 6 contains the conclusions.

\section{Integer programming formulations}

In Section 2.1, we give the IP-formulation for the single machine problem. In Section 2.2, we show how this formulation can be generalized to multiple machines.

\subsection{The single machine formulation}

Consider the problem of scheduling $n$ jobs on a single machine within a given timespan. The timespan $[0, T]$ is discretized into $T$ time periods of length one. Period $t$ refers to the time slot $[t-1, t] ; t=1, \ldots, T$. The processing time of job $j$ equals $p_{j}$. The machine can handle at most one job at a time and preemption is not allowed. When job $j$ starts in time period $t$, a known cost of $c_{j t}$ is incurred. The problem is to find a schedule that minimizes total cost.

This problem can be modeled as follows: for each job $j$ and for each time period $t=$ $1, \ldots, T$, we define

$$
x_{j t}= \begin{cases}1 & \text { if the processing of job } j \text { starts in time period } t \\ 0 & \text { otherwise. }\end{cases}
$$

The well-known time-indexed formulation for the single machine scheduling problem (as presented in Sousa and Wolsey (1992) and van den Akker et al. (1999)) is the following:

$$
\min \sum_{j=1}^{n} \sum_{t=1}^{T} c_{j t} x_{j t}
$$

subject to

$$
\begin{array}{cr}
\sum_{t=1}^{T} x_{j t}=1 & \forall j=1, \ldots, n, \\
\sum_{j=1}^{n} \sum_{s=t-p_{j}+1}^{t} x_{j s} \leq 1 & \forall t=1, \ldots, T, \\
x_{j t} \in\{0,1\} & \forall j=1, \ldots, n ; \forall t=1, \ldots, T .
\end{array}
$$


The objective function (1) minimizes the total cost. Constraints (2) state that each job has to be scheduled exactly once and constraints (3) express that during each time period $t$, only one job can be executed; we refer to (3) as the capacity constraints. This formulation is often called pseudo-polynomial because the number of variables and the number of constraints depend on the length of the time horizon. Thus, indeed if processing times are large, the number of variables grows. However, notice that (1) the problem is already strongly NP-hard if $p_{j}=2$ for all $j$ (Crama and Spieksma 1996) and (2) there exists applications where the cost of starting a job is 'truly' arbitrary, see e.g. the assignment of feeders to a component placement machine (Crama et al. 1990) or the assignment of ships to berths in container terminals (Hansen et al. 2008).

When one wants to generalize this formulation for the identical parallel machine scheduling problem, the right-hand side of constraints (3) can be set to $m$, the number of machines. However, when the machines are not identical, i.e., when a job's processing time depends on the machines, such a trick is not longer possible.

\subsection{Unrelated parallel machine scheduling}

We now consider the problem of scheduling $n$ jobs on $m$ unrelated parallel machines within a given timespan. Again, each machine can handle at most one job at a time and preemption is not allowed. The processing time of a job now depends on the machine: the processing time of job $i$ on machine $k$ is denoted by $p_{i k}$. The processing cost of a job depends both on the machine and the time period in which the job is started: the processing cost of job $i$ when executed at machine $k$ and started at time period $t$ is denoted by $c_{i k t}$. Again, we are interested in a feasible schedule minimizing total cost.

Unrelated parallel machine scheduling has received quite some attention in literature, especially the special case where one wants to minimize total weighted completion time. We will not review this literature, we simply mention Lenstra et al. (1990) and Gairing et al. (2007) and the references contained in those papers. Also, Vredeveld and Hurkens (2002) present an empirical comparison of different polynomial-time approximation algorithms and local search heuristics for the problem of minimizing total weighted completion time on unrelated parallel machines. The algorithms are based on rounding a fractional solution to an LP-relaxation or to a convex quadratic-programming relaxation.

We will model this unrelated parallel machine scheduling problem as a single machine problem in the following way: by copying each job $m$ times, we obtain $n m$ tasks $j$. We define $J$ as the set containing all tasks. This set can be partitioned in two different ways. First of all, we consider the subsets $J_{i} \subseteq J$ with $i=1, \ldots, n$ containing all tasks related to job $i$. Secondly, we consider the subsets $J^{k} \subseteq J$ with $k=1, \ldots, m$ containing all tasks related to machine $k$. Every subset $J_{i} \cap J^{k}$ consists of a single task $j$. The processing time of task $j=J_{i} \cap J^{k}$ equals $p_{j}=p_{i k}$. We denote by $c_{j t}=c_{i k t}$ the cost of starting task $j=J_{i} \cap J^{k}$ in time period $t$. Notice that specifying the task (index $j$ ), implies specifying the job and the machine, and vice versa..

For each task $j$ and for each time period $t=1, \ldots, T$, we define the decision variables

$$
x_{j t}= \begin{cases}1 & \text { if task } j \text { starts in time period } t \\ 0 & \text { otherwise }\end{cases}
$$


An IP-model for this machine scheduling problem is the following:

$$
\min \sum_{j=1}^{n m} \sum_{t=1}^{T} c_{j t} x_{j t}
$$

subject to

$$
\begin{array}{rr}
\sum_{j \in J_{i}} \sum_{t=1}^{T} x_{j t}=1 & \forall i=1, \ldots, n, \\
\sum_{j \in J^{k}} \sum_{s=t-p_{j}+1}^{t} x_{j s} \leq 1 & \forall k=1, \ldots, m ; \forall t=1, \ldots, T, \\
x_{j t} \in\{0,1\} & \forall j=1, \ldots, n m ; \forall t=1, \ldots, T .
\end{array}
$$

The objective function (5) minimizes the total cost. Constraints (6) state that out of the tasks related to job $i$, i.e., $J_{i}$, exactly one task has to be scheduled. The capacity constraints are formulated using constraint (7): for each time period $t$, only one task out of the tasks related to machine $k$, i.e., $J^{k}$, can be executed.

Notice that, in the case of a single machine, i.e. when $m=1$, formulation (5)-(8) becomes (1)-(4). In the remainder of this text, we will use $P_{m}$ to denote the convex hull of feasible solutions of (6)-(8).

\section{Known valid inequalities}

In this section, we review the known valid inequalities for $P_{1}$. Notice that an inequality for $P_{1}$ can be extended to an inequality for $P_{m}(m>1)$ by setting all coefficients that correspond to variables that involve tasks not related to some specific machine $k(1 \leq k \leq m)$ to 0 . Then, it is not difficult to observe that in this way, any inequality valid for $P_{1}$ can be extended to an inequality valid for $P_{m}$. We record this observation formally:

Fact 1 Any inequality valid for $P_{r}$ is valid for $P_{m}$, for each $r \leq m$.

Proof: We argue by contradiction. Suppose there is an inequality valid for $P_{r}$ which - when extended - is not valid for $P_{m}$. Hence, a feasible solution to the $m$-machine problem is cut off by the valid inequality. However, a feasible solution to an instance of the $m$-machine problem, when restricted to a subset of $r$ machines, becomes a feasible solution to an instance of the $r$-machine problem. Thus, we have identified a feasible solution to the $r$-machine problem that is cut off by the extended inequality, and hence also by the original inequality. This contradicts the initial assumption.

Fact 1 motivates us to formulate the known inequalities in terms of $P_{m}$. To do so, we need the following notation. For each $j=1, \ldots, n m$, we define $T(j)$ as the set of tasks that are related to the same machine as task $j$. Notice that $T(j)$ does not include task $j$. Moreover, we define $p_{j}^{*}=\max _{l \in T(j)} p_{l}$; thus $p_{j}^{*}$ is the largest processing time of the tasks in $T(j)$. 
Sousa and Wolsey (1992) give the following inequalities. For each time period $t=$ $1, \ldots, T$, for each task $j=1, \ldots, n m$ and for each $\Delta \in\left\{2, \ldots, p_{j}^{*}\right\}$ :

$$
\sum_{s=t-p_{j}+1}^{t+\Delta-1} x_{j s}+\sum_{l \in T(j)} \sum_{s=t-p_{l}+\Delta}^{t} x_{l s} \leq 1
$$

In inequality (9), task $j$ is sometimes called the 'special' task. These inequalities are known to be facet-defining for $P_{1}$ (Sousa and Wolsey 1992), and in fact they constitute all facet-defining inequalities for $P_{1}$ with right-hand side 1, (see van den Akker et al. 1999).

To give a pictorial description of this inequality, we will use a similar notation as van den Akker et al. (1999). The index-set of variables with nonzero coefficients in an inequality is denoted by $V$. The set of nonzero coefficients in an inequality associated with task $j$ defines a set of time periods $V_{j}=\{t \mid(j, t) \in V\}$. Thus the union over all $j$ of all $V_{j}$ equals $V$. We define an interval $[a, b]$ as the set of periods $\{a, a+1, \ldots, b\}$. If $a>b$, then $[a, b]=\emptyset$. We shall represent inequalities by diagrams. A diagram contains a line for each task. The blocks on the line associated with task $j$ indicate the time periods $t$ for which $x_{j t}$ occurs in the inequality.

Inequalities (9) of Sousa and Wolsey (1992) use the following time periods:

for task $j: \quad V_{j}=\left[t-p_{j}+1, t+\Delta-1\right]$,

for each task $l \in T(j): \quad V_{l}=\left[t-p_{l}+\Delta, t\right]$,

where $\Delta \in\left\{2, \ldots, p_{j}^{*}\right\}$.

These inequalities can be represented by the following diagram.

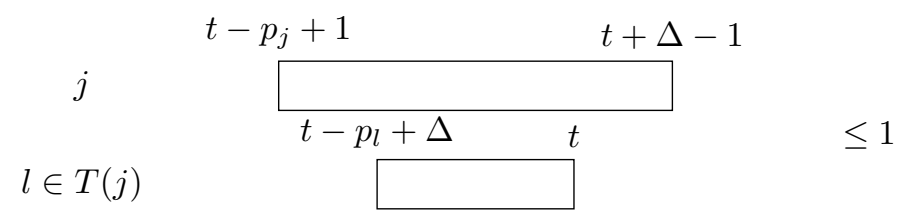

Using this diagram, it is relatively easy to see that inequalities (9) are valid. Indeed, notice that if some task $l \in T(j)$ starts at some time in $V_{l}$, no other task from $T(j)$ can start in $V_{l}$ (since both tasks would be active at time $t$ ). Also task $j$ cannot start in $V_{j}$, since starting task $j$ directly after the completion of task $l$ is impossible: task $l$ is active until $t+\Delta-1$; starting task $j$ before the beginning of task $l$ is equally impossible, since even starting task $j$ at $t-p_{j}+1$ means that task $j$ is active at time $t$. This implies validity of (9).

van den Akker et al. (1999) present three classes of valid inequalities with integral coefficients and right-hand side 2. We sketch informally how these classes can be constructed using two valid inequalities (9), one with $j=j^{\prime}$ for $t=t_{1}$ (the left one), and one with $j=j^{\prime \prime} \in T\left(j^{\prime}\right) \cup\left\{j^{\prime}\right\}$ for $t=t_{2}>t_{1}$ (the right one). Choose two additional tasks $j^{*} \in T\left(j^{\prime}\right)$ and $j^{* *} \in T\left(j^{\prime \prime}\right)$. For each task $l \in T\left(j^{\prime}\right)$ with $l \neq j^{*}$, we decrease $V_{l}$ of the left inequality by changing its first element $t_{1}-p_{l}+\Delta$ to $t_{1}-p_{l}+\Delta+u+1$. Similarly, for each task $l \in T\left(j^{\prime \prime}\right)$ with $l \neq j^{* *}$, we decrease $V_{l}$ of the right inequality by changing its last element $t_{2}$ to $t_{2}-v$. As a consequence, we can add some additional coefficients in between both 
sets. We compose the sets $V_{j}$ of these inequalities as follows $V_{j}=L_{j} \cup M_{j} \cup U_{j}$. $L_{j}$ and $U_{j}$ refer to the inequalities (9) while $M_{j}$ contains the additional coefficients. Variables that are in $L_{j} \cap U_{j}$ will have coefficient 2 in the valid inequality. For the first class of inequalities, $j_{1}=j^{\prime}=j^{\prime \prime}$ and $j_{2}=j^{*}=j^{* *}$. For the second class $j_{1}=j^{\prime}=j^{\prime \prime}, j_{2}=j^{*}$ and $j_{3}=j^{* *}$. Finally, for the third class of inequalities, $j_{1}=j^{\prime}=j^{* *}$ and $j_{2}=j^{\prime \prime}=j^{*}$.

In order to specify the first class of inequalities, we consider the following intervals. For all time periods $t_{1}, t_{2}=1, \ldots, T$, for all tasks $j_{1}=1, \ldots, n m, j_{2} \in T\left(j_{1}\right)$, for all $\Delta_{1}, \Delta_{2} \in\left\{2, \ldots, p_{j_{1}}^{*}\right\}$ and for all $-1 \leq u, v \leq t_{2}-t_{1}-\Delta_{1}$,

$$
\begin{aligned}
& L_{j_{1}}=\left[t_{1}-p_{j_{1}}+1, t_{1}+\Delta_{1}-1\right], \quad U_{j_{1}}=\left[t_{2}-p_{j_{1}}+1, t_{2}+\Delta_{2}-1\right], \\
& M_{j_{1}}=\left[t_{2}-p_{j_{1}}-v, t_{1}+\Delta_{1}+u\right] \backslash\left(L_{j_{1}} \cup U_{j_{1}}\right), \\
& L_{j_{2}}=\left[t_{1}-p_{j_{2}}+\Delta_{1}, t_{1}\right], \quad U_{j_{2}}=\left[t_{2}-p_{j_{2}}+\Delta_{2}, t_{2}\right], \\
& M_{j_{2}}=\left[\max \left\{t_{2}-v, t_{1}+\Delta_{1}\right\}-p_{j_{2}}, \min \left\{t_{1}+\Delta_{1}+u, t_{2}\right\}\right] \backslash\left(L_{j_{2}} \cup U_{j_{2}}\right),
\end{aligned}
$$

for each task $l \in T\left(j_{1}\right) \backslash\left\{j_{2}\right\}$ :

$$
\begin{aligned}
& L_{l}=\left[t_{1}-p_{l}+\Delta_{1}+u+1, t_{1}\right], \quad U_{l}=\left[t_{2}-p_{l}+\Delta_{2}, t_{2}-v-1\right], \\
& M_{l}=\left[t_{2}-p_{l}+1, t_{1}+\Delta_{1}-1\right] \backslash\left(L_{l} \cup U_{l}\right) .
\end{aligned}
$$

These inequalities can be represented by the diagram presented in Figure 1.

In order to specify the second class of inequalities, we consider the following intervals. For all time periods $t_{1}, t_{2}=1, \ldots, T$, for all tasks $j_{1}=1, \ldots, n m,\left\{j_{2}, j_{3}\right\} \in T\left(j_{1}\right)$, for all $\Delta_{1}, \Delta_{2} \in\left\{2, \ldots, p_{j_{1}}^{*}\right\}$ and for all $u, v \geq-1$ with $u+v \leq t_{2}-t_{1}-\Delta_{1}$ and either $u>-1$ or $v>-1$,

$$
\begin{aligned}
& L_{j_{1}}=\left[t_{1}-p_{j_{1}}+1, t_{1}+\Delta_{1}-1\right], \quad U_{j_{1}}=\left[t_{2}-p_{j_{1}}+1, t_{2}+\Delta_{2}-1\right], \\
& M_{j_{1}}=\emptyset, \\
& L_{j_{2}}=\left[t_{1}-p_{j_{2}}+\Delta_{1}, t_{1}\right], \quad U_{j_{2}}=\left[t_{2}-p_{j_{2}}+\Delta_{2}, t_{2}-v-1\right], \\
& M_{j_{2}}=\left[t_{2}-p_{j_{2}}+1, t_{1}+\Delta_{1}+u\right] \backslash\left(L_{j_{2}} \cup U_{j_{2}}\right), \\
& L_{j_{3}}=\left[t_{1}-p_{j_{3}}+\Delta_{1}+u+1, t_{1}\right], \quad U_{j_{3}}=\left[t_{2}-p_{j_{3}}+\Delta_{2}, t_{2}\right], \\
& M_{j_{3}}=\left[t_{2}-p_{j_{3}}-v, t_{1}+\Delta_{1}-1\right] \backslash\left(L_{j_{3}} \cup U_{j_{3}}\right), \\
& \text { for each task } l \in T\left(j_{1}\right) \backslash\left\{j_{2}, j_{3}\right\}: \\
& L_{l}=\left[t_{1}-p_{l}+\Delta_{1}+u+1, t_{1}\right], \quad U_{l}=\left[t_{2}-p_{l}+\Delta_{2}, t_{2}-v-1\right], \\
& M_{l}=\left[t_{2}-p_{l}+1, t_{1}+\Delta_{1}-1\right] \backslash\left(L_{l} \cup U_{l}\right) .
\end{aligned}
$$

These inequalities can be represented by the diagram presented in Figure 2.

Finally, in order to specify the third class of inequalities, we consider the following intervals. For all time periods $t_{1}, t_{2}=1, \ldots, T$, for all tasks $j_{1}=1, \ldots, n m, j_{2} \in T\left(j_{1}\right)$, for all $\Delta_{1} \in\left\{1, \ldots, p_{j_{1}}^{*}\right\}$, for all $\Delta_{2} \in\left\{1, \ldots, p_{j_{2}}^{*}\right\}$ and for all $-1 \leq u, v$,

$$
\begin{aligned}
& L_{j_{1}}=\left[t_{1}-p_{j_{1}}+1, t_{1}+\Delta_{1}-1\right], \quad U_{j_{1}}=\left[t_{2}-p_{j_{1}}+\Delta_{2}, t_{2}\right], \\
& M_{j_{1}}=\left[t_{2}-p_{j_{1}}-v, \min \left\{t_{1}+\Delta_{1}+u, t_{2}\right\}\right] \backslash\left(L_{j_{1}} \cup U_{j_{1}}\right), \\
& L_{j_{2}}=\left[t_{1}-p_{j_{2}}+\Delta_{1}, t_{1}\right], \quad U_{j_{2}}=\left[t_{2}-p_{j_{2}}+1, t_{2}+\Delta_{2}-1\right], \\
& M_{j_{2}}=\left[\max \left\{t_{2}-v, t_{1}+\Delta_{1}\right\}-p_{j_{2}}, t_{1}+\Delta_{1}+u\right] \backslash\left(L_{j_{2}} \cup U_{j_{2}}\right), \\
& \text { for each task } l \in T\left(j_{1}\right) \backslash\left\{j_{2}\right\}:
\end{aligned}
$$$$
L_{l}=\left[t_{1}-p_{l}+\Delta_{1}+u+1, t_{1}\right], \quad U_{l}=\left[t_{2}-p_{l}+\Delta_{2}, t_{2}-v-1\right] \text {, }
$$$$
M_{l}=\emptyset
$$ 


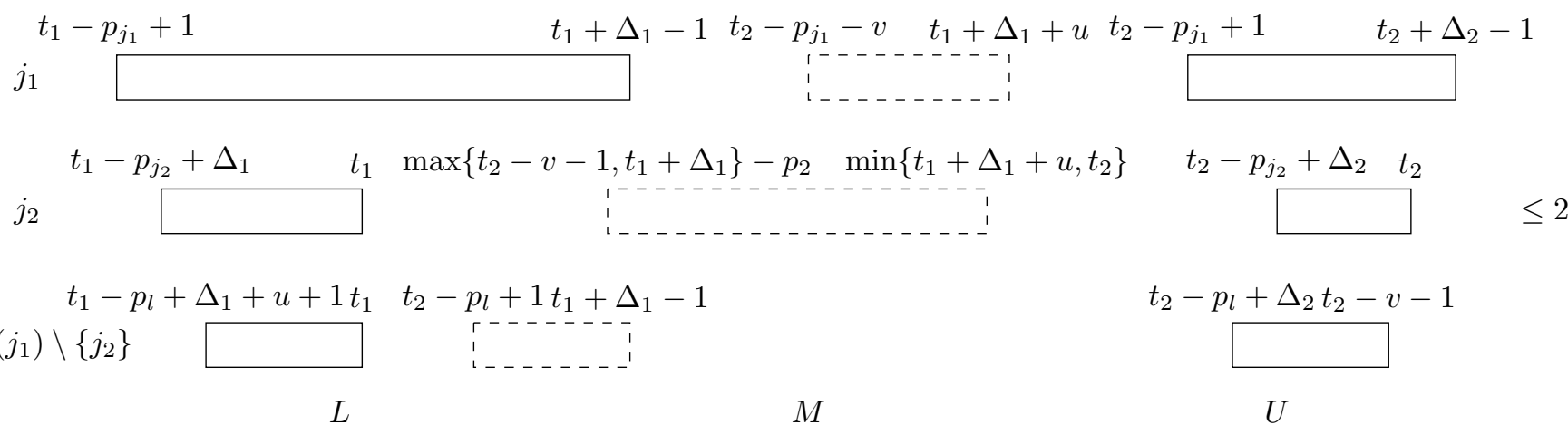

Figure 1: The diagram representing the first set of extended valid inequalities. 


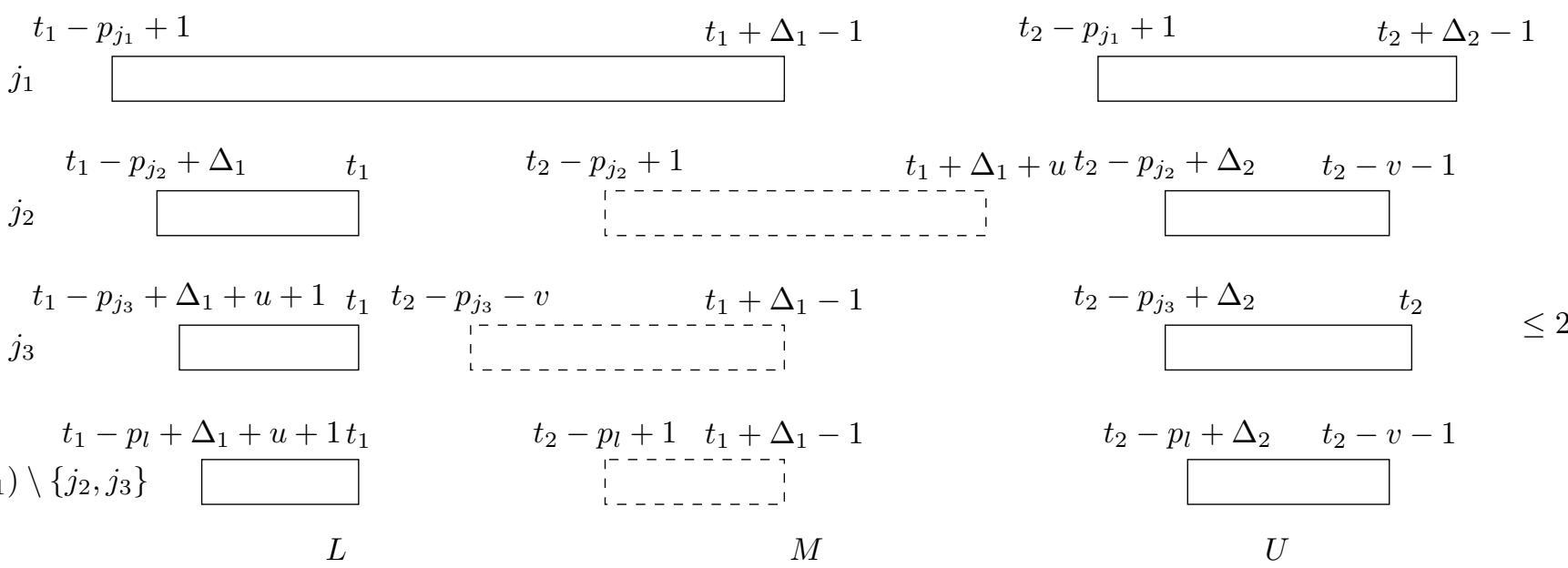

Figure 2: The diagram representing the second set of extended valid inequalities. 


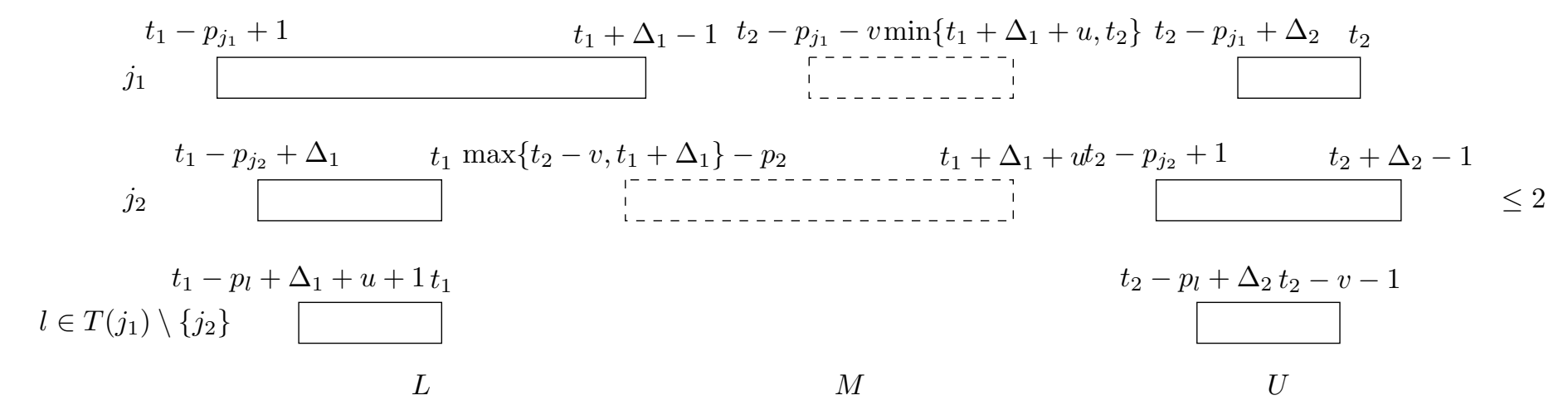

0

Figure 3: The diagram representing the third set of extended valid inequalities. 
These inequalities can be represented by the diagram presented in Figure 3.

A general notation for the three sets of inequalities is the following: for all tasks $j_{1}=$ $1, \ldots, n m$ :

$$
\sum_{l \in T\left(j_{1}\right) \cup\left\{j_{1}\right\}}\left(\sum_{t \in L_{l}} x_{l t}+\sum_{t \in M_{l}} x_{l t}+\sum_{t \in U_{l}} x_{l t}\right) \leq 2
$$

It is proven in van den Akker et al. (1999) that inequalities (10) are all facet-defining inequalities with right-hand side 2 for $P_{1}$.

\section{Inequalities}

In this section, we introduce a new class of valid inequalities that contains variables corresponding to different machines.

\subsection{Example}

We now specify an instance. Let $n=3, m=2, T=14, J^{1}=\{1,2,3\}, J^{2}=\{4,5,6\}$, $J_{1}=\{1,4\}, J_{2}=\{2,5\}, J_{3}=\{3,6\}, p_{1}=4, p_{2}=3, p_{3}=5, p_{4}=1, p_{5}=5$ and $p_{6}=2$, Further, the $c_{j t}$ coefficients are given in Table 1 where the tasks can be found on the vertical axis, and where the time periods can be found on the horizontal axis.

\begin{tabular}{|l|lllllllllllllll|}
\hline & 0 & 1 & 2 & 3 & 4 & 5 & 6 & 7 & 8 & 9 & 10 & 11 & 12 & 13 & 14 \\
\hline 1 & 2 & 2 & 2 & 2 & 1 & 2 & 2 & 2 & 2 & 2 & 2 & 2 & 2 & 2 & 2 \\
2 & 1 & 1 & 1 & 1 & 1 & 1 & 1 & 1 & 0 & 1 & 1 & 1 & 1 & 1 & 1 \\
3 & 1 & 1 & 1 & 1 & 1 & 1 & 1 & 0 & 1 & 1 & 1 & 1 & 1 & 1 & 1 \\
4 & 2 & 2 & 2 & 2 & 2 & 1 & 2 & 2 & 2 & 2 & 2 & 2 & 2 & 2 & 2 \\
5 & 1 & 0 & 1 & 1 & 1 & 1 & 1 & 1 & 1 & 1 & 1 & 1 & 1 & 1 & 1 \\
6 & 1 & 1 & 1 & 1 & 1 & 1 & 1 & 1 & 1 & 1 & 1 & 1 & 1 & 1 & 1 \\
\hline
\end{tabular}

Table 1: The coefficients $c_{j t}$ for the example instance.

The LP-relaxation (5) - (8) of this instance is the fractional solution $x_{1,4}=x_{2,8}=x_{3,7}=$ $x_{4,5}=x_{5,1}=x_{6,2}=\frac{1}{2}$. This solution is not cut off neither by (9) nor by (10). This is a fact since the sum of all variables on one machine is $<2$. We will now describe a class of valid inequalities that are able to cut away this fractional solution.

\subsection{A new class of valid inequalities}

For each pair of jobs $\left\{i, i^{\prime}\right\} \in\{1, \ldots, n\}$, and for each pair of machines $\left\{k, k^{\prime}\right\} \in\{1, \ldots, m\}$, let $j=J^{k} \cap J_{i}, q=J^{k} \cap J_{i^{\prime}}, a=J^{k^{\prime}} \cap J_{i}$ and $b=J^{k^{\prime}} \cap J_{i^{\prime}}$. For each quadruple of such four tasks, for all time periods $t_{1}, t_{2}=1, \ldots, T$, for all $\Delta_{1} \in\left\{1, \ldots, \min \left\{p_{j}^{*}, p_{q}^{*}\right\}\right\}$ and for all 


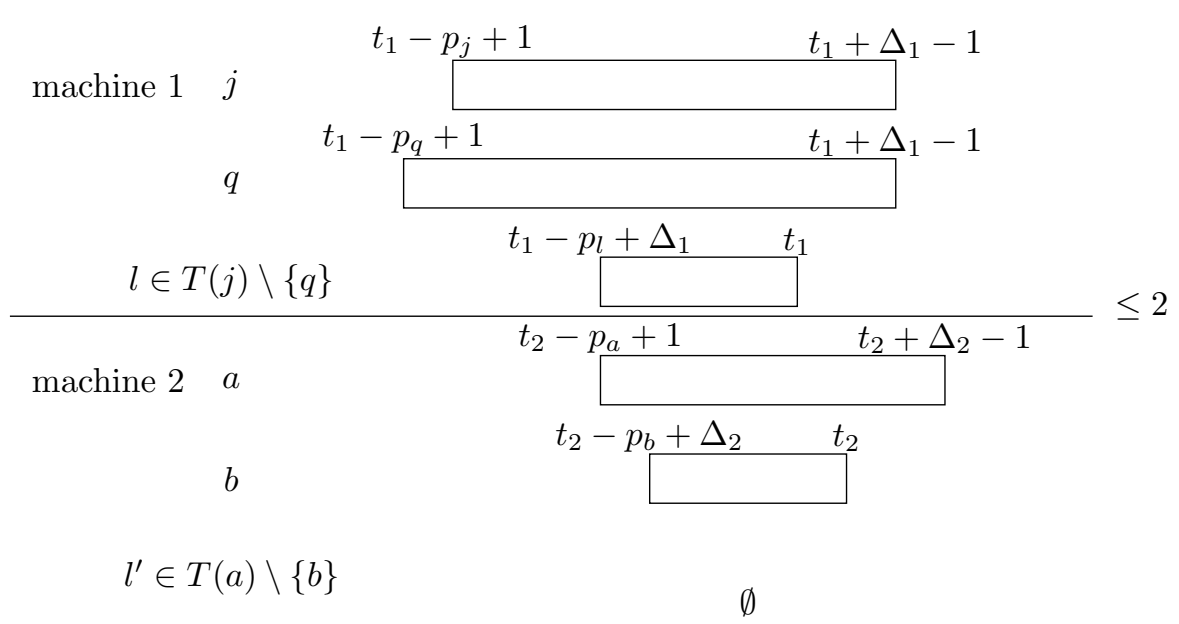

Figure 4: The diagram representing the valid inequalities (11).

$\Delta_{2} \in\left\{1, \ldots, p_{a}^{*}\right\}$, we have the following inequalities:

$$
\begin{array}{r}
\sum_{s=t_{1}-p_{j}+1}^{t_{1}+\Delta_{1}-1} x_{j s}+\sum_{s=t_{1}-p_{q}+1}^{t_{1}+\Delta_{1}-1} x_{q s}+\sum_{l \in T(j) \backslash\{q\}} \sum_{s=t_{1}-p_{l}+\Delta_{1}}^{t_{1}} x_{l s} \\
+\sum_{s=t_{2}-p_{a}+1}^{t_{2}+\Delta_{2}-1} x_{a s}+\sum_{s=t_{2}-p_{b}+\Delta_{2}}^{t_{2}} x_{b s} \leq 2
\end{array}
$$

These inequalities can be represented by the diagram presented in Figure 4 (where we take $k=1$, and $k^{\prime}=2$ ).

Lemma 2 Inequalities (11) are valid inequalities for $P_{m}$, for each $m \geq 2$.

Proof: We will show that any feasible solution satisfies (11). Remark that it is not possible to start the two tasks $a$ and $b$ during the considered time interval as the capacity for period $t_{2}$ would be violated. We distinguish the following three cases.

Case 1: No task starts on machine 2 during the considered time intervals. Only tasks related to the first machine will be scheduled during the considered time intervals.

Case 1a: We start both tasks $j$ and $q$. Each of these tasks occupies at least one of the periods $\left\{t_{1}, \ldots, t_{1}+\Delta_{1}-1\right\}$. Since each task in $l \in T(j) \backslash\{q\}$ occupies all these time periods when started in the interval $\left[t_{1}-p_{l}+\Delta_{1}, t_{1}\right]$, it is impossible to start a third task.

Case 1b: We start either task $j$ or task $q$. At least one of the periods in the interval $\left\{t_{1}, \ldots, t_{1}+\Delta_{1}-1\right\}$ will be occupied. And as each task $l \in T(j) \backslash\{q\}$ occupies all these periods when started in the interval $\left[t_{1}-p_{l}+\Delta, t_{1}\right]$, only one of them can be started in the considered intervals.

Case 1c: We do not start tasks $j$ and $q$. As each task $l \in T(j) \backslash\{q\}$ occupies periods $t_{1}$ when started in the interval $\left[t_{1}-p_{l}+\Delta, t_{1}\right]$, only one of them can be started in the considered intervals.

Case 2: Task $a$ on machine 2 is started during the considered time intervals. This implies 
that we cannot start task $j$ since tasks $j$ and $a$ related to the same job.

Case 2a: We start task $q$ at any period of the considered interval $\left[t_{1}-p_{q}+1, t_{1}+\Delta_{1}-1\right]$. We cannot start another task $l \in T(j)$ as starting such a task in the interval $\left[t_{1}-p_{l}+\Delta_{1}, t_{1}\right]$ will occupy all time periods $\left\{t_{1}, \ldots, t_{1}+\Delta_{1}-1\right\}$ and at least one of these periods is taken by task $q$.

Case 2b: We do not start task $q$, and we only start tasks from $l \in T(j) \backslash\{q\}$. From all these tasks, we can start only one during the considered time interval $\left[t_{1}-p_{l}+\Delta_{1}, t_{1}\right]$, since otherwise the capacity for period $t_{1}$ would be violated.

Case 3: Task $b$ on machine 2 is started during the considered time intervals. This implies that we cannot start task $q$ in the considered interval because for each job only one task can be started.

Case 3a: We start task $j$ at any period of the considered interval $\left[t_{1}-p_{j}+1, t_{1}+\Delta_{1}-1\right]$. Then, we cannot start another task $l \in T(j)$ as starting such a task in the interval $\left[t_{1}-p_{l}+\Delta_{1}, t_{1}\right]$ will occupy all time periods $\left\{t_{1}, \ldots, t_{1}+\Delta_{1}-1\right\}$ and at least one of these periods is taken by task $j$.

Case 3b: We do not start task $j$, and we only start tasks from $l \in T(j) \backslash\{q\}$. From all these tasks, we can start only one during the considered time interval $\left[t_{1}-p_{l}+\Delta_{1}, t_{1}\right]$, since otherwise the capacity for period $t_{1}$ would be violated.

\subsection{Example continued}

With $j=1, q=2, a=4, b=5, t_{1}=7, \Delta_{1}=4, t_{2}=4$ and $\Delta_{2}=2$ this inequality (11) boils down to

$$
\begin{aligned}
x_{1,4} & +x_{1,5}+x_{1,6}+x_{1,7}+x_{1,8}+x_{1,9}+x_{1,10}+x_{2,5}+x_{2,6}+x_{2,7}+x_{2,8} \\
& +x_{2,9}+x_{2,10}+x_{3,6}+x_{3,7}+x_{4,4}+x_{4,5}+x_{5,1}+x_{5,2}+x_{5,3}+x_{5,4} \leq 2 .
\end{aligned}
$$

It is displayed by the squared blocks in the example and cuts off the fractional solution.

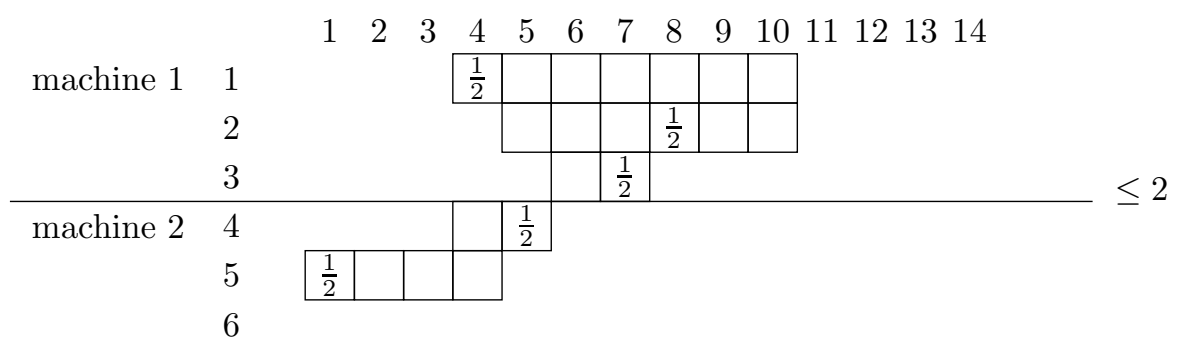

\section{Computational results}

In this section we describe our implementation of a basic cutting-plane algorithm (Section 5.1 ), we give details concerning the generation of our instances (Section 5.2), and we present and discuss our computational results (Section 5.3). 


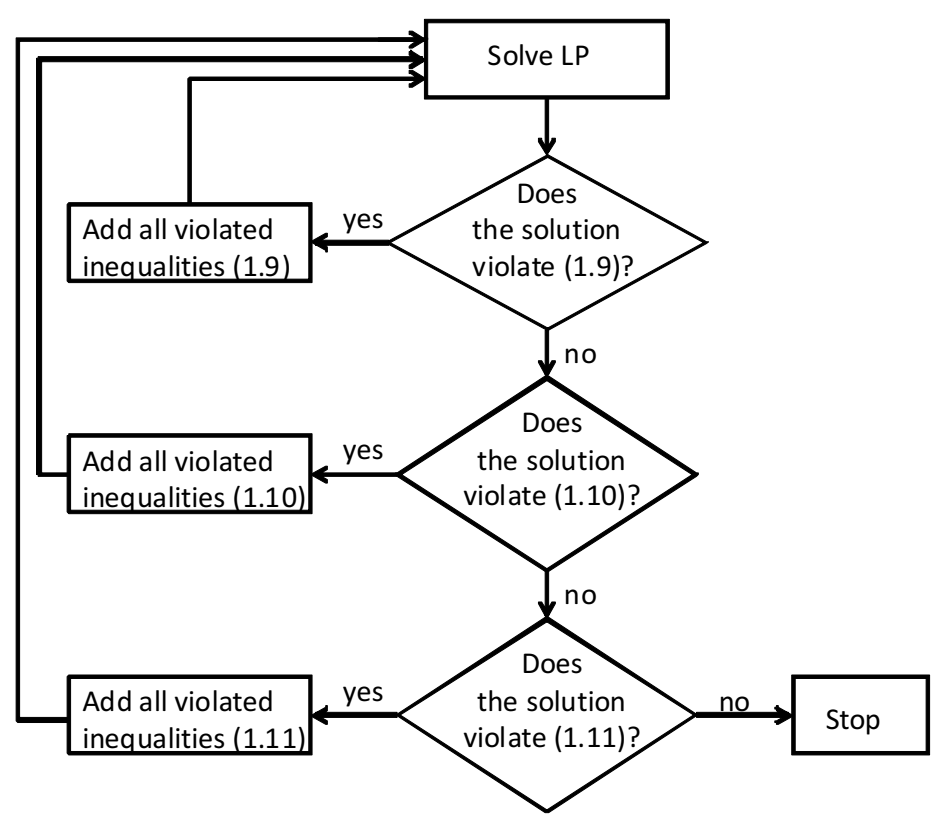

Figure 5: The basic cutting-plane algorithm

\subsection{Separation}

We have implemented a basic cutting-plane algorithm. Its working is illustrated in Figure 5. The separation algorithm basically enumerates all possible valid inequalities of a specific type and tests whether it is violated. Its operation is similar to the separation algorithm described in van den Akker et al. (1999). Notice that the vector $\tilde{x}$, the current LP-solution, will be sparse; this fact is used in the separation. The separation algorithm for inequalities (11) is explicitly given below.

\subsection{Generation instances}

We created instances in line with Sousa and Wolsey (1992) and van den Akker et al. (1999) in the following way: the number of jobs $n \in\{20,30,40\}$, the number of machines $m \in$ $\{1,2,3,5,10\}$. The time horizon is defined as $|T|=3.1 \frac{n}{m}$. The maximal processing time is $p_{\max }=5$ and the processing times on both machines are uniformly distributed in $\left[1, p_{\max }\right]$. Finally, the processing costs $c_{j t}$ on all machines are uniformly distributed in $[1,100]$. All data are integral. For each combination of $n$ and $m, 20$ instances were created, yielding 300 instances in total.

We define $|T|$ proportional to $\frac{n}{m}$ to have the same average number of possible starting times for each task over all instances. In preliminary results, we tested different choices for $|T|$ and different intervals for $c_{j t}$. It turned out that with $c_{j t} \in[1,10]$, the resulting instances were easier to solve than the current instances: the LP gap was smaller and the LP solutions were more often integral. Since we want instances with a large gap, the settings presented above were chosen. 


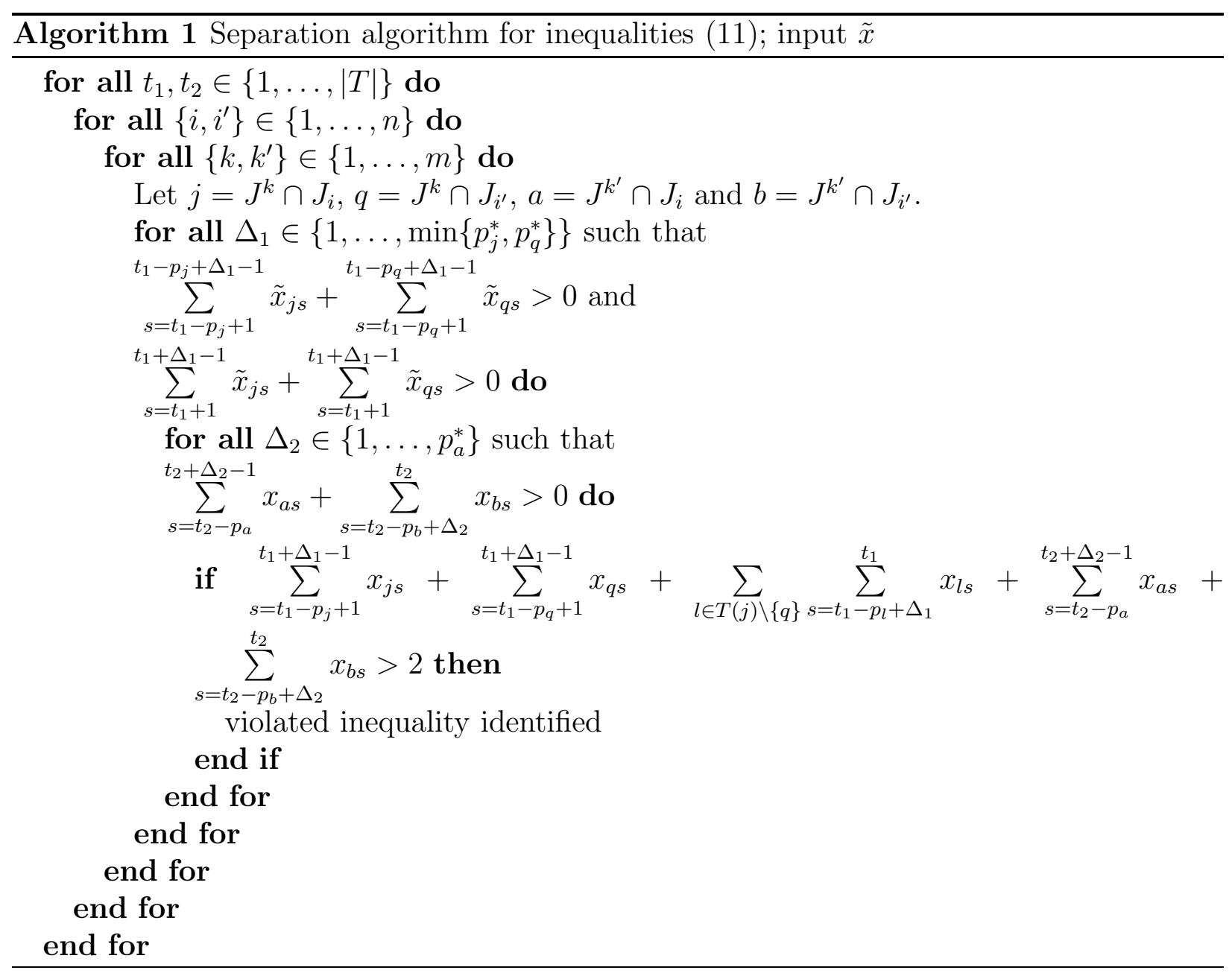


Table 2: Number of instances that were solved to optimality

\begin{tabular}{|l|llll|}
\hline$(m \times n)$ & $n_{L P}$ & $n_{(9)}$ & $n_{(10)}$ & $n_{(11)}$ \\
\hline $1 \times 20$ & 7 & 1 & 1 & \\
$1 \times 30$ & 7 & 0 & 1 & \\
$1 \times 40$ & 1 & 1 & 0 & \\
\hline $2 \times 20$ & 6 & 5 & 1 & 0 \\
$2 \times 30$ & 10 & 2 & 0 & 0 \\
$2 \times 40$ & 11 & 0 & 0 & 2 \\
\hline $3 \times 20$ & 8 & 3 & 0 & 1 \\
$3 \times 30$ & 11 & 1 & 0 & 1 \\
$3 \times 40$ & 7 & 0 & 0 & 1 \\
\hline $5 \times 20$ & 12 & 3 & 0 & 0 \\
$5 \times 30$ & 8 & 5 & 0 & 4 \\
$5 \times 40$ & 9 & 1 & 1 & 1 \\
\hline $10 \times 20$ & 16 & 1 & 0 & 0 \\
$10 \times 30$ & 10 & 1 & 0 & 1 \\
$10 \times 40$ & 12 & 2 & 0 & 1 \\
\hline
\end{tabular}

\subsection{Results}

All algorithms are encoded in C using the Microsoft Visual Studio programming environment, and executed on a Dell Latitude D630 with an Intel Pentium-4 2.2-GHz processor and 2 GB RAM, equipped with Windows 7. CPLEX version 10.2 is used to solve the IP and LP models.

Since our primary goal is to see to what extent our valid inequalities help bridging the gap between $z_{L P}$ and $z_{I P}$, we do not report precise running times. Instead, we restrict ourselves here to some general remarks. Even for large instances, computing $z_{I P}$ takes up to a few seconds, while computing $z_{L P}$ takes some fractions of a second. Running the separation algorithm generally takes up to a few minutes. The number of iterations is rarely larger than five; we come back to this issue in Table 4.

Table 2 provides statistics on the frequency with which optimal solutions are found. More precisely, we report the number of instances for which the optimal solution of the LP-relaxation is integral (see column $n_{L P}$ ), the number of instances for which the solution becomes integral after the addition of cuts (9) ( see column $\left.n_{(9)}\right)$, after the addition of cuts (10) ( see column $n_{(10)}$ ), and after addition of cuts (11) (see column $\left.n_{(11)}\right)$. We see that a significant portion of the instances has an integral LP-solution, to be precise $45 \%$. This percentage seems to grow mildly with the size of an instance. Adding inequalities (9) helps in producing integral solutions: another 26 out of the 300 instances become integral. Inequalities (10) are less successful, while inequalities (11) yield another 12 instances. Indeed, the percentage of integral solutions increases with the number of machines.

In Table 3, we focus on the instances whose LP-relaxation is fractional, column '\#' indicates the number of those instances. We present the average value of the LP solution 
Table 3: Average percentage of the gap that is closed after adding valid inequalities

\begin{tabular}{|l|l||l|l|l|l|l|}
\hline$(m \times n)$ & $\#$ & $z_{L P}$ & $(9)$ & $(10)$ & $(11)$ & $z_{I P}$ \\
\hline $1 \times 20$ & 13 & 66.22 & $25.74 \%$ & $39.53 \%$ & & 68.77 \\
$1 \times 30$ & 13 & 72.69 & $19.27 \%$ & $21.37 \%$ & & 74.69 \\
$1 \times 40$ & 19 & 79.50 & $31.83 \%$ & $37.24 \%$ & & 81.42 \\
\hline $2 \times 20$ & 14 & 70.11 & $61.04 \%$ & $61.37 \%$ & $68.29 \%$ & 72.07 \\
$2 \times 30$ & 10 & 68.16 & $36.55 \%$ & $41.37 \%$ & $45.69 \%$ & 69.70 \\
$2 \times 40$ & 9 & 73.91 & $7.66 \%$ & $7.66 \%$ & $41.76 \%$ & 74.89 \\
\hline $3 \times 20$ & 12 & 81.04 & $44.21 \%$ & $55.00 \%$ & $68.11 \%$ & 82.17 \\
$3 \times 30$ & 9 & 69.81 & $52.89 \%$ & $52.89 \%$ & $59.74 \%$ & 70.90 \\
$3 \times 40$ & 13 & 73.73 & $20.52 \%$ & $26.13 \%$ & $35.82 \%$ & 74.62 \\
\hline $5 \times 20$ & 8 & 65.81 & $41.67 \%$ & $41.67 \%$ & $58.32 \%$ & 67.13 \\
$5 \times 30$ & 12 & 71.11 & $52.01 \%$ & $52.01 \%$ & $80.84 \%$ & 71.58 \\
$5 \times 40$ & 11 & 76.28 & $4.55 \%$ & $13.64 \%$ & $44.51 \%$ & 76.91 \\
\hline $10 \times 20$ & 4 & 62.06 & $35.71 \%$ & $35.71 \%$ & $35.71 \%$ & 62.75 \\
$10 \times 30$ & 10 & 67.55 & $10.00 \%$ & $10.00 \%$ & $40.00 \%$ & 68.00 \\
$10 \times 40$ & 8 & 76.29 & $17.51 \%$ & $22.93 \%$ & $43.09 \%$ & 77.00 \\
\hline
\end{tabular}

as $z_{L P}$ and the average value of the IP solution as $z_{I P}$. Moreover, the percentage of the gap $\left(z_{I P}-z_{L P}\right)$ that is closed after adding valid inequalities is displayed. The percentage is computed as $100 \times \frac{z(\tilde{x})-z_{L P}}{z_{I P}-z_{L P}}$ where $z(\tilde{x})$ is the value found after adding the corresponding inequalities. When, in an extreme case, the LP solution and the IP solution have the same value, although the LP solution is fractional, we say that the gap is closed with $0 \%$ if the solution stays fractional after adding cuts and the gap is closed with $100 \%$ when the solution becomes integral. We conclude that each of the three classes has a contribution in closing (part of) the gap. Inequalities (9) are quite powerful, bridging on average $30.74 \%$ of the gap. Inequalities (10) contribute an additional percentage to half of the instance sets. Inequalities (11) are quite effective, bridging an additional $17.26 \%$ of the gap.

In Table 4, we also focus on the instances whose LP-relaxation is fractional. We report the overall ('Total') number of iterations ('\# it') and the overall number of added valid inequalities ('\# VI'). Each time that we solve the LP-relaxation including added valid inequalities, we count one iteration. So the first time we solve the LP-relaxation (before adding inequalities) does not count as an iteration. Moreover, we report how many iterations give rise to adding valid inequalities of a certain set and how many valid inequalities were found and added. We see that on average, the cutting plane algorithm is stopped after 2.05 iterations, either because an integral solution is obtained or because no more violated inequalities can be found. The number of added valid inequalities is on average 7.58. Very few iterations add valid inequalities of type (10). When inequalities of type (11) are added, the number of inequalities added is on average 6.49 inequalities, which is large compared to 1 for inequalities (9) and 1.45 for inequalities (10).

Tables 3 and 4 allow us to conclude that inequalities 11 are useful in a cutting-plane approach for solving instances of unrelated parallel machine scheduling problems. 
Table 4: Average number of iterations and number of adding valid inequalities

\begin{tabular}{|l||ll|ll|ll|ll|}
\hline \multicolumn{1}{|c||}{$(m \times n)$} & \multicolumn{2}{c|}{$(9)$} & \multicolumn{2}{c|}{$(10)$} & \multicolumn{2}{c|}{$(11)$} & \multicolumn{2}{c|}{ Total } \\
& \# it & \# VI & \# it & \# VI & \# it & \# VI & \# it & \# VI \\
\hline $1 \times 20$ & 0.77 & 0.92 & 0.23 & 2.00 & & & 1.85 & 2.92 \\
$1 \times 30$ & 0.77 & 1.00 & 0.38 & 9.38 & & & 2.08 & 10.38 \\
$1 \times 40$ & 0.79 & 1.21 & 0.58 & 3.89 & & & 2.32 & 5.11 \\
\hline $2 \times 20$ & 1.07 & 1.36 & 0.21 & 1.50 & 0.43 & 9.36 & 2.29 & 12.21 \\
$2 \times 30$ & 1.10 & 1.70 & 0.30 & 1.80 & 0.40 & 7.00 & 2.70 & 9.60 \\
$2 \times 40$ & 0.78 & 1.11 & 0.00 & 0.00 & 0.67 & 4.22 & 2.22 & 5.33 \\
\hline $3 \times 20$ & 1.00 & 1.58 & 0.25 & 1.25 & 0.75 & 5.67 & 2.67 & 8.50 \\
$3 \times 30$ & 1.20 & 1.50 & 0.00 & 0.00 & 0.60 & 7.20 & 2.60 & 8.70 \\
$3 \times 40$ & 0.77 & 1.08 & 0.15 & 1.00 & 0.54 & 8.46 & 2.38 & 10.54 \\
\hline $5 \times 20$ & 0.88 & 1.00 & 0.00 & 0.00 & 0.38 & 4.75 & 1.88 & 5.75 \\
$5 \times 30$ & 0.67 & 0.75 & 0.00 & 0.00 & 0.42 & 5.92 & 1.25 & 6.67 \\
$5 \times 40$ & 0.18 & 0.27 & 0.09 & 0.91 & 0.73 & 12.00 & 1.82 & 13.18 \\
\hline $10 \times 20$ & 0.50 & 0.50 & 0.00 & 0.00 & 0.00 & 0.00 & 1.25 & 0.50 \\
$10 \times 30$ & 0.20 & 0.20 & 0.00 & 0.00 & 0.30 & 3.10 & 1.30 & 3.30 \\
$10 \times 40$ & 0.63 & 0.75 & 0.00 & 0.00 & 0.75 & 10.25 & 2.13 & 11.00 \\
\hline
\end{tabular}

\section{Conclusion}

We modeled the unrelated parallel machine scheduling problem where the processing cost of each job is an arbitrary function of its starting time as a single machine scheduling problem using a time-indexed formulation. We have shown that valid inequalities from literature for single-machine problems can be applied to multi-machine problems. A new set of inequalities is presented and a cutting plane algorithm is proposed. We have shown the computational performance of this algorithm by testing it on randomly generated instances.

\section{References}

L. Bigras, M. Gamache, and G. Savard. Time-indexed formulations and the total weighted tardiness problem. INFORMS Journal on Computing, 20(1):133-142, 2008.

Y. Crama and F. C. R. Spieksma. Scheduling jobs of equal length: Complexity, facets and computational results. Mathematical Programming, 72:207-227, 1996.

Y. Crama, A. W. J. Kolen, A. G. Oerlemans, and F. C. R. Spieksma. Throughput rate optimization in the automated assembly of printed circuit boards. Annals of Operations Research, 26:455-480, 1990.

M. E. Dyer and L. A. Wolsey. Formulating the single machine sequencing problem with release dates as a mixed integer problem. Discrete Applied Mathematics, 26:255-270, 1990. 
M. Gairing, B. Monien, and A. Woclaw. A faster combinatorial approximation algorithm for scheduling unrelated parallel machines. Theoretical Computer Science, 380:87-99, 2007.

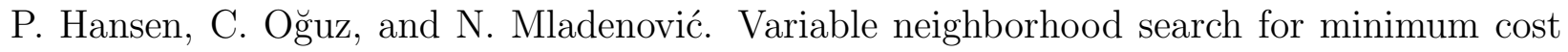
berth allocation. European Journal of Operational Research, 191(3):636-649, 2008.

J. K. Lenstra, D. B. Shmoys, and E. Tardos. Approximation algorithms for scheduling unrelated parallel machines. Mathematical Programming, 46:259-271, 1990.

F. Sourd. New exact algorithms for one-machine earliness-tardiness scheduling. INFORMS Jounal of Computing, 21:167-175, 2009.

J. P. Sousa and L. A. Wolsey. A time indexed formulation of non-preemptive single machine scheduling problems. Mathematical Programming, 54:353-367, 1992.

S. Tanaka, S. Fujikuma, and M. Araki. An exact algorithm for single-machine scheduling without machine idle time. Journal of Scheduling, 12:575-593, 2009.

Y. Unlu and S. J. Mason. Evaluation of mixed integer programming formulations for nonpreemptive parallel machine scheduling problems. Computers $\&$ Industrial Engineering, 58:785-800, 2010.

J. M. van den Akker, J. A. Hoogeveen, and S. L. van de Velde. Parallel machine scheduling by column generation. Operations Research, 47(6):862-872, 1999.

J. M. van den Akker, C. A. J. Hurkens, and M. W. P. Savelsbergh. Time-indexed formulations for machine scheduling problems: Column generation. INFORMS Journal on Computing, 12(2):111-124, 2000.

J.M. van den Akker, C.P.M. Van Hoesel, and M.W.P. Savelsbergh. A polyhedral approach to single-machine scheduling problems. Mathematical Programming, 85:541-572, 1999.

T. Vredeveld and C. Hurkens. Experimental comparison of approximation algorithms for scheduling unrelated parallel machines. INFORMS Journal on Computing, 14(2):175-189, 2002. 